\title{
Search for the Standard Model Higgs boson at CMS
}

\author{
Abdollah Mohammadi ${ }^{1, a}$, on behalf of the CMS Collaboration \\ ${ }^{1}$ IIHE-ULB, Universite Libre de Bruxelle, Brussels, Belgium
}

\begin{abstract}
A search has been performed for the Standard Model (SM) Higgs boson in different production modes and decay channels. The results are based on up to $5 \mathrm{fb}^{-1}$ of $7 \mathrm{TeV}$ and up to $20 \mathrm{fb}^{-1}$ of $8 \mathrm{TeV}$ proton-proton collisions data accumulated by the CMS experiment at LHC in 2011 and 2012. Additionally several tests have been done to check the compatibility of the observed state with the SM Higgs boson.
\end{abstract}

\section{Introduction}

One of the main goals of the LHC physics program is to ascertain the mechanism of electroweak symmetry breaking, through which the $\mathrm{W}$ and $\mathrm{Z}$ bosons attain mass. In the SM, this is achieved via the Brout-Englert-Higgs mechanism, which also predicts the existence of a scalar Higgs boson. The Yukawa coupling of the Higgs field to fermions generates fermion masses. In the SM, the Higgs boson is produced via different production modes. The highest cross-section production belongs to the gluon-gluon Fusion ( $\mathrm{ggH}$ ) followed by the Vector Boson Fusion (VBF) which is characterised by the presence of 2 jets in the forward direction. The other main production mechanisms are Higgs boson production in association with a $\mathrm{W}$ or $\mathrm{Z}$ boson and finally production with $\mathrm{t} \overline{\mathrm{t}}$. At the low masses the most important Higgs decay modes in fermionic channels are bb and $\tau \tau$, and in bosonic channels are WW, ZZ and $\gamma \gamma$. In July 2012, both CMS and ATLAS experiments at the Large Hadron Collider announced the observation of a significant excess of events on top of the SM backgrounds which is consistent with the presence of such a scalar boson with a mass around $125 \mathrm{GeV}[1,2]$. In this paper, using the full data from the CMS Run 1, all production and decay modes have been investigated. A precise measurement of the Higgs boson mass is presented. Additionally several tests have been performed to check the compatibility of the observed state with the SM Higgs boson.

\section{CMS Detector}

The central feature of the CMS apparatus is a superconducting solenoid of $6 \mathrm{~m}$ internal diameter, providing a magnetic field of $3.8 \mathrm{~T}$. Within the superconducting solenoid volume are a silicon pixel and strip tracker, a lead tungstate crystal electromagnetic calorimeter (ECAL), and a brass and scintillator hadron calorimeter (HCAL), each composed of a barrel and two endcap sections. Muons are detected in gas-ionization detectors embedded in the steel flux-return yoke outside the solenoid. Extensive forward calorimetry complements the coverage provided by the barrel and endcap detectors. A more

ae-mail: abdollah.mohammadi@cern.ch 
detailed description of the CMS detector, together with a definition of the coordinate system used and the relevant kinematic variables, can be found in Ref. [3].

The first level of CMS triggering system (L1), composed of custom hardware processes, uses information from calorimeters and muons systems, and decreases the initial $40 \mathrm{MHz}$ collision rate to about $100 \mathrm{kHz}$. The high level trigger (HLT) processor farm, exploiting the full information of various subdectors, reduces the rate further to about $300 \mathrm{~Hz}$ before storing the data on tape.

\section{Event reconstruction}

The event reconstruction follows the particle-flow (PF) algorithm, which aims at exploiting all the information from all subdetectors to identify individual particles, such as charged hadrons, neutral hadrons, muons, electrons or photons. Complex objects, such as taus, jets and missing transverse energy $\mathrm{E}_{\mathrm{T}}^{\mathrm{miss}}$, are reconstructed from these individual particles.

Muon reconstruction starts from matching the silicon tracker tracks to standalone-muon tracks (tracks from muon spectrometer). A global muon track is fitted combining hits from the tracker tracks and standalone-muon tracks. Several quality cuts are applied on muon track and muon impact parameter to distinguish real prompt muons from spurious muons or muons coming from cosmic rays. Additionally, muons are required to pass isolation criteria to separate the prompt muons from those coming within a jet, usually from the semi-leptonic decay of heavy quarks.

Electron reconstruction starts from ECAL superclusters, which are groups of one or more associated clusters of energy deposit in the ECAL. Superclusters are matched to track seeds in the inner tracker layers and electron tracks are built from those. Trajectories are reconstructed based on the modelling of electron energy loss due to electron radiation and are fitted with the Gaussian Sum Filter (GSF) algorithm. Electron identification is based on a multivariate technique Boosted Decision Tree (BDT) to discriminate real electrons from jets faking electrons. The main handles for such discrimination are the ratio of energy deposits in the ECAL and HCAL, as well as the angular difference between track and supercluster, and finally electron shower shape. Similar to muons, PFlow based isolation is used to select the real prompt electrons from the ones within a jet.

Photon candidates are reconstructed from energy deposits in the ECAL in a similar way to electrons. The clustering algorithm takes into account photon conversion and Bremsstrahlung in the material in front of the ECAL. A BDT is trained to distinguish prompt photons from the photon candidates from mis-identification of the jets. Several variables are used as inputs to the BDT including lateral shower shape, isolation variables, the energy median density per unit area in the event, the pseudorapidity and supercluster energy.

The Hadron Plus Strips (HPS) algorithm is used to reconstruct the hadronic decays of taus. It starts from a PF jet with a cone size of $\Delta R=0.5$ and searches for tau lepton decay candidates produced by any of the hadronic decay modes of the tau lepton. Taus decay hadronically either directly to one charged hadron or indirectly via intermediate $\rho$ and a1 mesons to one charged hadron plus one to two neutral pions and three charged hadrons and always accompanied by a neutrino. In most tau decays to charged hadrons, some neutral pions are produced and decay instantly to a pair of photons. While attempting to combine charged hadrons and photons in ECAL 'strips' to reconstruct various decay modes, the HPS algorithm takes into account the broadening of the energy deposits from photons in the magnetic field direction in the ECAL. Taus are required to satisfy the isolation criteria and not to be matched to electrons and muons to reduce electron and muon mis-identification rates, respectively.

$\mathrm{PF}$ jets are reconstructed using the anti-kt algorithm with a cone size of $\Delta R=0.5$. Several corrections are applied to the jet energy to reduce the pileup (multiple interaction per bunch crossing) effect. Jets are required to pass a multivariate discriminator to reject jets coming from pile-up. Jets originating from a b-quark (b-jets) are identified with the Combined Secondary Vertex (CSV) algorithm, 
which is based on a likelihood technique and exploits all known parameters to distinguish b-jets from jets not originating from b-quarks such as impact parameter significance, presence of a secondary vertex and jet kinematics.

Finally, missing transverse energy, $\overrightarrow{\mathrm{E}}_{\mathrm{T}}^{\mathrm{miss}}$, is the negative of the vector sum of the transverse momenta of all final state particles reconstructed in the detector.

\section{$4 \mathbf{H} \rightarrow \gamma \gamma$}

In spite of the small branching fraction of the Higgs boson to a pair of photons, clean signature and high di-photon mass resolution lead this channel to be one of the most sensitive ones. Different production modes are exploited in this analysis and are tagged based on the presence of extra jet pair (either forward or non-forward), electron, muon or missing transverse energy. Untagged events are further categorised according to a BDT score that aims at separating categories with higher signal over background ratio from those with lower signal over BG ratio using photon identification, isolation criteria, shower shape and mass resolution as inputs. Main backgrounds are either due to the prompt non-resonant di-photon events or events with at least one jet misidentified as an isolated photon. To suppress events from $\mathrm{Z} \rightarrow \mathrm{e}^{+} \mathrm{e}^{-}$events, an electron veto is applied to remove the photon candidates if they are matched to reconstructed electrons.

For each production mode and category, a parametric signal model is constructed from a fit of the simulated invariant mass shape. For the background, a fit has been performed on the $\mathrm{m}_{\gamma \gamma}$ distribution in data, without reference to the MC simulation. Figure 1-left shows the $\mathrm{m}_{\gamma \gamma}$ distribution combining all categories for combined 7 and $8 \mathrm{TeV}$ data. A clear excess of events is observed. The Higgs boson mass is found to be $124.70 \pm 0.34 \mathrm{GeV}$. The plot on the right shows the local p-values and significance of this excess. The observed local significance is $5.7 \sigma$, where a significance of $5.2 \sigma$ is expected for the standard model Higgs boson [4].
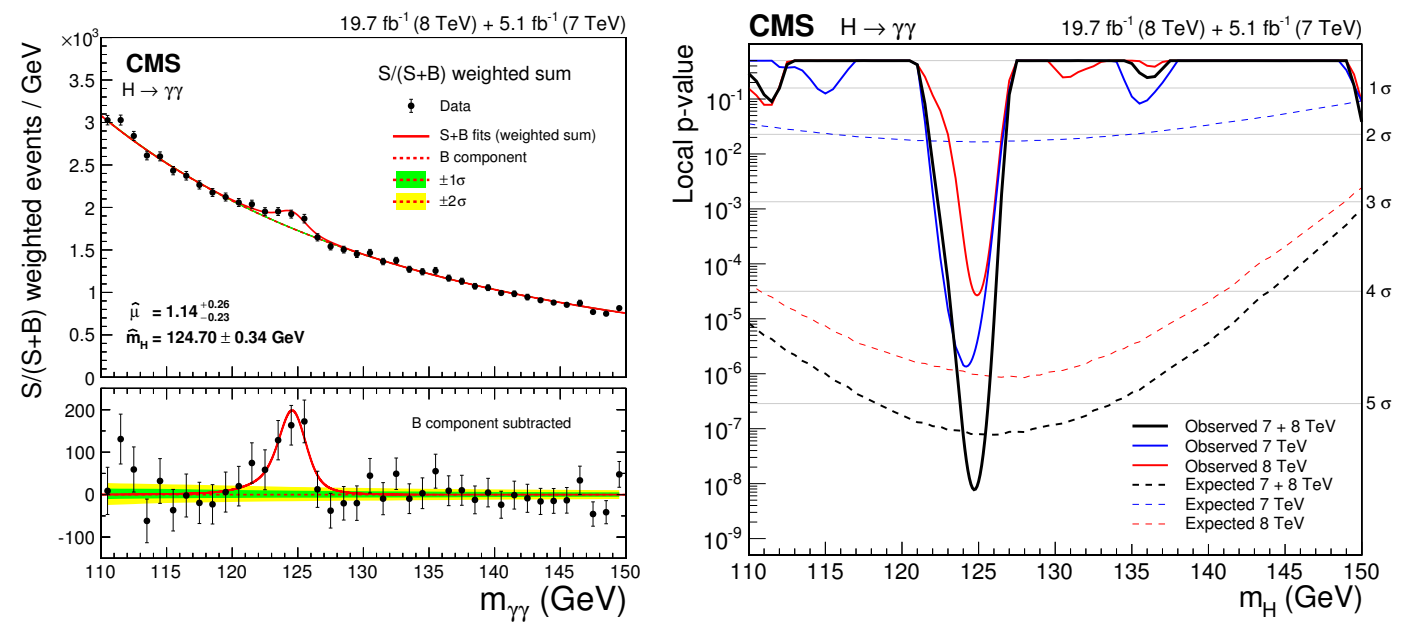

Figure 1. $\mathrm{m}_{y \gamma}$ distribution combining all production modes and different categories for combined 7 and $8 \mathrm{TeV}$ data(left), observed and expected significance corresponding to the signal in the di-photon channel. 


\section{$5 \mathrm{H} \rightarrow \mathrm{ZZ}$}

Referred usually as the "Golden Channel", $\mathrm{ZZ} \rightarrow 41$, is the cleanest channel among all the decay modes of the Higgs boson. The unique signature of this mode consists of the presence of 4 identified and isolated leptons $(4 \mu, 4 \mathrm{e}$ or $2 \mu 2 \mathrm{e})$ triggered online using di-lepton or tri-lepton trigger. While the first $\mathrm{Z}$ is required to fulfil mass requirement between 40 and $120 \mathrm{GeV}$, the second $\mathrm{Z}$ mass is allowed to be between 12 and $120 \mathrm{GeV}$. The main background in this channel is the irreducible non-resonant $\mathrm{ZZ}$ production with both $\mathrm{Z}$ boson decay leptonically leading to 4 isolated leptons and is directly estimated from MC simulation. Additionally, there are some reducible backgrounds, like $t \bar{t}, \mathrm{WZ}+\mathrm{jet}$ and $\mathrm{Z}+\mathrm{jet}$ which are estimated from data. In order to increase the sensitivity to the different production modes, events are split into two categories. Events with 2 jets are tagged as 'di-jet' while other events stand in 'untagged' category.

An event-by-event estimator of the mass resolution has been built from the single-lepton momentum resolutions evaluated from the study of $J / \psi \rightarrow \mu \mu$ and $\mathrm{Z} \rightarrow$ ll data events. Mass of the 4 lepton $m_{4 l}$, a kinematical discriminant $D_{\mathrm{bkg}}^{\mathrm{kin}}$ (which is calculated using each $\mathrm{Z}$ boson mass and 5 angles in the $\mathrm{H}$ and $\mathrm{Z}$ decay plane) and the $\mathrm{p}_{\mathrm{T}}$ of 4 lepton $\mathrm{p}_{\mathrm{T}}{ }^{4 l}$ ( $D_{\text {jet }}$ for 'di-jet' tagged events) are used for statistical interpretation.

Figure 2-left shows the $m_{4 l}$ distribution for combined 7 and $8 \mathrm{TeV}$ data. The Higgs signal is observed on top of the relatively flat background around the mass of $125 \mathrm{GeV}$. This excess is equivalent to the local observed(expected) significance of 6.9(6.6) $\sigma$ which is shown in Figure 2-right [5].
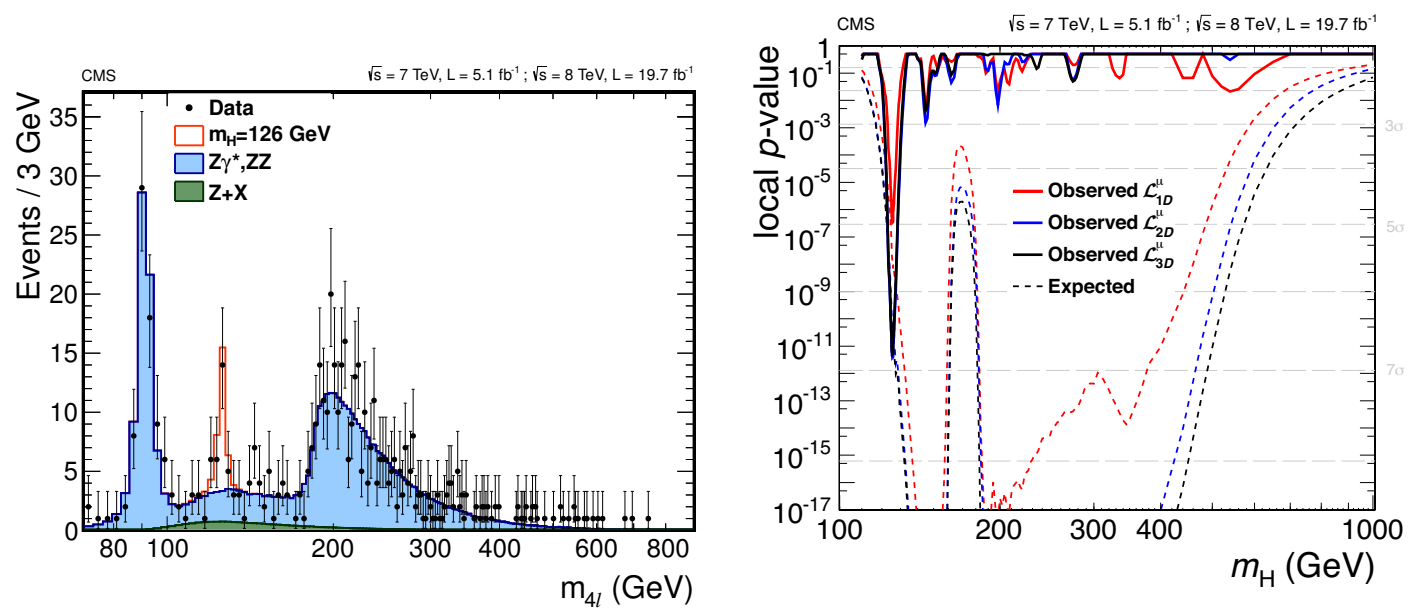

Figure 2. $m_{4 l}$ distribution in the $\mathrm{ZZ} \rightarrow 41$ final state (left) and significance of the local excess with respect to the SM background expectation as a function of the Higgs boson mass for the $1 \mathrm{D}$ fit $\left(\mathcal{L}_{1 D}^{\mu}=\mathcal{L}_{1 D}^{\mu}\left(m_{4 l}\right)\right)$, 2D fit $\left(\mathcal{L}_{2 D}^{\mu}=\mathcal{L}_{2 D}^{\mu}\left(m_{4 l}, D_{\mathrm{bkg}}^{\mathrm{kin}}\right)\right)$, and the nominal 3D fit $\left(\mathcal{L}_{3 D}^{\mu}=\mathcal{L}_{3 D}^{\mu}\left(m_{4 l}, D_{\mathrm{bkg}}^{\mathrm{kin}}, \mathrm{p}_{\mathrm{T}}{ }^{4 l}\right)\right.$ (right).

\section{$6 \mathrm{H} \rightarrow \mathrm{WW}$}

Due to the presence of neutrinos in the decay of $\mathrm{W}$ to leptons in $\mathrm{WW} \rightarrow 212 v$ final state, it is not possible to observe a peak in the di-lepton mass distribution, instead we are looking for an excess of events on top of the SM backgrounds. Different production modes considering 2 leptons, or 3 leptons 
and moderate missing transverse energy and multiple jets are exploited to increase the sensitivity of different production modes. Events with two leptons are split into 6 categories with different background composition and signal over background ratios, from 3 different possible pairs of electron and muon and either 0 or 1 jet. Events with 2 forward jets are tagged as VBF production mode. If those 2 jets do not satisfy the VBF criteria, events are tagged as VH. In all categories, WW di-boson production is an important and irreducible background and its normalisation and shape are estimated from MC simulation. In order to suppress the overwhelming DY background in same flavor (ee or $\mu \mu$ ) channels, the invariant mass of the lepton pair is required to be far from $\mathrm{Z}$ boson mass (i.e. 76 to $106 \mathrm{GeV}$ ). Apart from DY background, other reducible ones are $\mathrm{t} \overline{\mathrm{t}}, \mathrm{QCD}$ and $\mathrm{W}+\mathrm{jets}$ which are estimated from data. The statistical interpretation in different flavor 0 and 1 jet events is based on the two dimensional distribution in the $\left(m_{l l}, m_{T}\right)$ plane, where $m_{l l}$ is the invariant mass of the di-lepton pair and $m_{T}$ is transverse mass constructed with the lepton pair and missing transverse energy. For the other categories, a counting experiment has been used for measurements.

In the $\mathrm{WH} \rightarrow \mathrm{WWW} \rightarrow 313 v$ final state, we are looking for an excess of events with three leptons. Each pair of same flavor opposite charge leptons must not be compatible with the leptonic decay of a $\mathrm{Z}$ boson. This requirement reduces the $\mathrm{WZ} \rightarrow 31 v$ background a lot. Other backgrounds such as $\mathrm{Z}+\mathrm{jets}$ and top quark production are estimated from data. To perform the measurement the smallest angular distance between the oppositely-charged lepton tracks has been used. The $\mathrm{ZH} \rightarrow \mathrm{ZWW} \rightarrow 31 v 2 \mathrm{j}$ is also used to tag events in the $\mathrm{ZH}$ production mode. Two same flavor opposite sign leptons compatible with $\mathrm{Z}$ boson decay and an extra lepton and moderate missing transverse energy from the leptonic decay of $\mathrm{W}$ boson plus 2 jets from hadronic decay of $\mathrm{W}$ boson $\left(\left|m_{j j}-m_{W}\right|<60 \mathrm{GeV}\right)$ are the signatures of this final state. The main backgrounds from $\mathrm{WZ}$ and $\mathrm{ZZ}$ and tri-bosons are estimated from $\mathrm{MC}$ simulation while the processes involving the non-prompt leptons are estimated from data. The transverse mass of the lvjj system is used as the observable to perform the measurement.
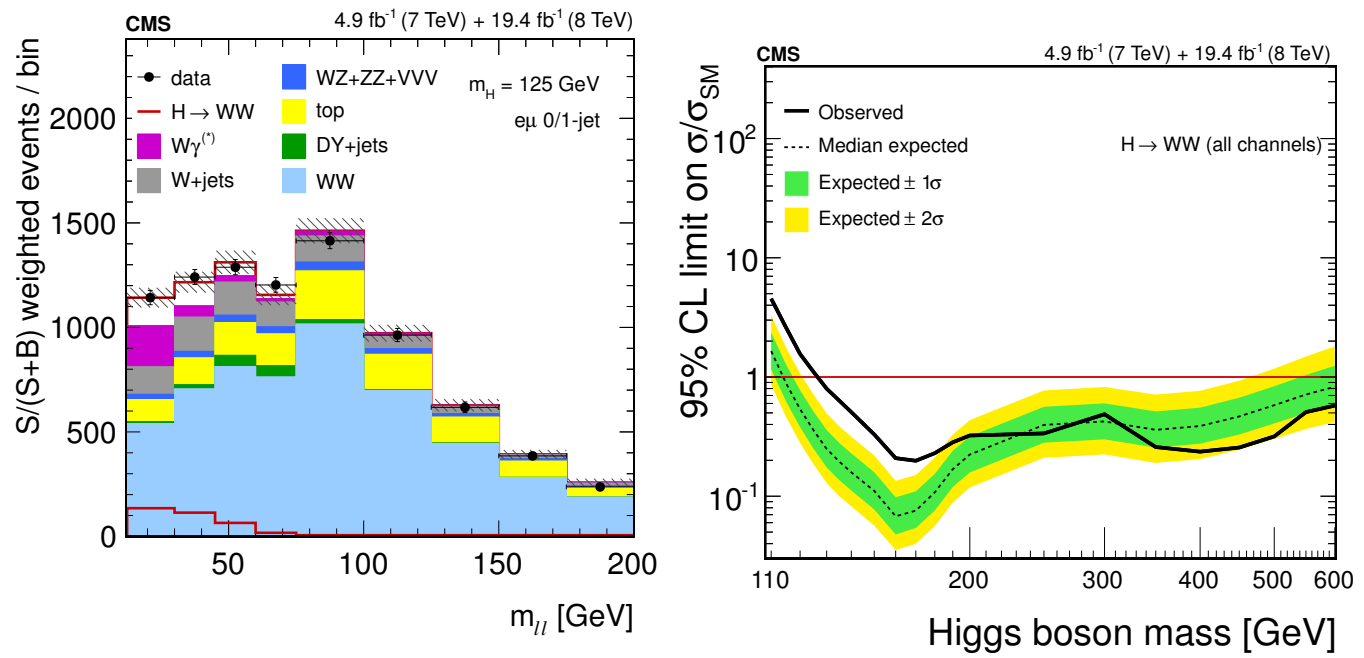

Figure 3. $m_{l l}$ distribution of the e $\mu$ channel in $0 / 1$ jet category (left) and expected and observed $95 \%$ CL upper limits on the Higgs boson production cross section in terms of a ratio to the SM prediction as a function of the Higgs boson mass (right). 
Figure 3-left shows the $m_{l l}$ distribution of one process (e $\mu$ in $0 / 1$ jet category). There is a large excess of events at low values of the $m_{l l}$ distribution which are not compatible with the SM backgrounds and completely compatible with the presence of the Higgs boson at $125 \mathrm{GeV}$. The right plot shows expected and observed 95\% CL upper limits on the Higgs boson production cross section in terms of a ratio to the SM prediction, as a function of the Higgs boson mass. The large difference between observed and expected limit indicates the incompatibility of the observed data with background-only model [6].

\section{$7 \mathbf{H} \rightarrow \tau \tau$}

The search for the SM Higgs boson to fermions is an important test for Yukawa coupling. $\mathrm{H} \rightarrow \tau \tau$ is the most promising channel for this test. The search is done for different production modes and all possible decay modes of the taus. Events are initially split into exclusive categories based on the number of leptons (electrons, muons or hadronic taus). Events with 4 leptons are targeted to explore the Higgs production in association with a $\mathrm{Z}$ boson decaying to a pair of electrons or muons. Events with three leptons are used to tag the $\mathrm{WH}$ production where the $\mathrm{W}$ decays leptonically to either an electron or a muon. Finally events with 2 leptons are used to tag gluon-gluon Fusion or VBF production modes. These events are further categorised based on the jet multiplicity to events with 0 , 1 or 2 jets.

In the $\mathrm{e} \tau_{\mathrm{h}}, \mu \tau_{\mathrm{h}}, \tau_{\mathrm{h}} \tau_{\mathrm{h}}$ and $\mathrm{e} \mu$ final state, the important irreducible background from $Z / \gamma * \rightarrow \tau \tau$ is modelled by using an embedding technique. In this technique, the reconstructed muons from the $Z / \gamma * \rightarrow \mu \mu$ events selected in data are replaced by generator level tau leptons. The taus are decayed using TAUOLA. The embedded samples are normalised according to the yield of $Z / \gamma * \rightarrow \mu \mu$ events measured in a control region. QCD is also a major background, especially at low di-tau mass. The contribution of the QCD multi-jet background arises from jet $\rightarrow \tau_{\mathrm{h}}$ mis-identification and to a lesser extent from jet $\rightarrow$ e mis-identification in e $\tau_{\mathrm{h}}$ final state. Normalisation of this background in $\mu \tau_{\mathrm{h}}$ and $e \tau_{\mathrm{h}}$ final states is measured in same sign (SS) di-lepton regions (which is orthogonal to the oppositesign (OS) signal region) times an OS/SS extrapolation factor. The shape of QCD background is estimated from data in a SS regions. W background is measured using the high $\mathrm{M}_{\mathrm{T}}$ sideband and a high $\mathrm{M}_{\mathrm{T}}$ to low $\mathrm{M}_{\mathrm{T}}$ extrapolation factor is estimated from MC. Other backgrounds, such as $Z / \gamma * \rightarrow \mu \mu$ and $Z / \gamma * \rightarrow e e, \mathrm{t} \bar{t}$, single top and di-boson (WW, WZ, ZZ) represent a small fraction of the total background in this analysis. Both shapes and normalisations are taken from MC simulations. In WH $(\mathrm{ZH})$ channels, the shape and normalisation of the irreducible background, WZ(ZZ), are estimated from MC simulation. The reducible background arising from $\mathrm{W}+$ jet, $\mathrm{Z}+$ jet and $\mathrm{tt}$ where at least one jet is misidentified as a lepton is estimated directly from data.

Due to the presence of neutrinos in the decay of the tau lepton, a likelihood approach is adopted to reconstruct the mass of di-tau system in $\mathrm{e} \tau_{\mathrm{h}}, \mu \tau_{\mathrm{h}}, \tau_{\mathrm{h}} \tau_{\mathrm{h}}, \mathrm{e} \mu$ and $\mathrm{ZH}$ channels. This reconstructed mass is used to perform the measurement. In channels tagged as $\mathrm{WH}$ due to extra neutrino from $\mathrm{W}$ decay, the visible mass of the Higgs decay products is used for measurement. In the ee and $\mu \mu$ final states, the search is performed using the combination of two MVA discriminants. The first is trained to distinguish $\mathrm{Z} \rightarrow$ ll events from $\mathrm{Z} \rightarrow \tau \tau$ events while the second to separate $\mathrm{Z} \rightarrow \tau \tau$ from $\mathrm{H} \rightarrow \tau \tau$ events. Figure 4-left shows the weighted sum of the $m_{\tau \tau}$ distributions for four final states. The excess of events on top of the SM is compatible with the one expected from SM. Combining all production modes and channels leads to the observed (expected) significance of 3.2(3.7) $\sigma$ at a mass of $125 \mathrm{GeV}$ shown in the right plot [7]. 

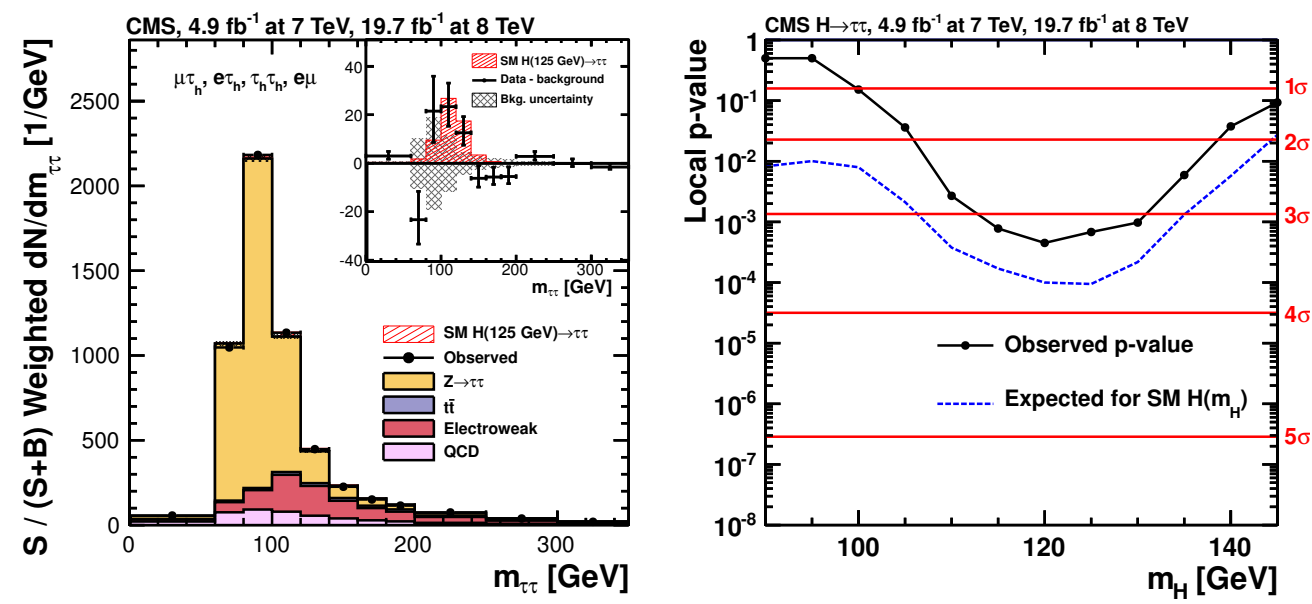

Figure 4. Weighted sum of the $m_{\tau \tau}$ distributions for $\mathrm{e} \tau_{\mathrm{h}}, \mu \tau_{\mathrm{h}}, \tau_{\mathrm{h}} \tau_{\mathrm{h}}$ and e $\mu$ final states (left), observed and expected significance for a combination all production and decay modes in $\tau \tau$ final state(right).

\section{$8 \mathrm{H} \rightarrow \mathrm{bb}$}

Amongst all possible decay of the SM Higgs boson at low masses, $\mathrm{H} \rightarrow$ bb has the largest branching fraction. However the search in gluon-gluon Fusion is extremely difficult due to the overwhelming multijet QCD background. The most sensitive channel is the search for the associated production of the Higgs boson with either a $\mathrm{W}$ or $\mathrm{Z}$ boson, where the $\mathrm{W}$ decays leptonically to either electron, muon or hadronic tau or the $\mathrm{Z}$ decays leptonically to a pair of electrons or muons or decays to neutrinos leading to missing transverse energy. Presence of a lepton or $E_{T}^{m i s s}$ suppress multijet QCD by a large factor. The event sample is divided into categories depending on the transverse momentum of the vector boson.

The Higgs boson candidate is reconstructed by requiring two b-tagged jets. An MVA regression is used to estimate the true $b$ quark energy after being trained on reconstructed $b$ jets in simulated $\mathrm{H} \rightarrow \mathrm{bb}$ events. To improve the sensitivity of signal, several MVA classifiers are exploited which have been trained to distinguish the signal from $\mathrm{V}+$ jet, di-boson and $\mathrm{t} \overline{\mathrm{t}}$ backgrounds. The normalisation of these backgrounds are estimated from data using signal free control regions. Other backgrounds, like WZ, $\mathrm{ZZ}$ and single top that involve real $\mathrm{b}$ jets, are estimated from MC simulation.

For the statistical interpretation the MVA classifier outputs are used for all production modes. This MVA method to extract the signal is validated on VZ di-boson which has exactly the same signature of the Higgs signal except that the two b-tagged jets are originated from the $\mathrm{Z}$ boson instead of the Higgs boson. This test led to the observation of an excess of events for the combined WZ and ZZ processes which differs by over 7 standard deviation from event yield from background only hypothesis. Figure 5-left shows the distribution of $\log$ of S (signal) over B (background). To make these plots, events are sorted in bins of similar expected signal-to-background ratio, as given by the value of the output of their corresponding BDT discriminant (trained with a Higgs boson mass hypothesis of 125 $\mathrm{GeV}$ ), combing all final states. The right plots shows the observed and expected significance. Both of them correspond to $2.0 \sigma$ at $125 \mathrm{GeV}$ [8]. 

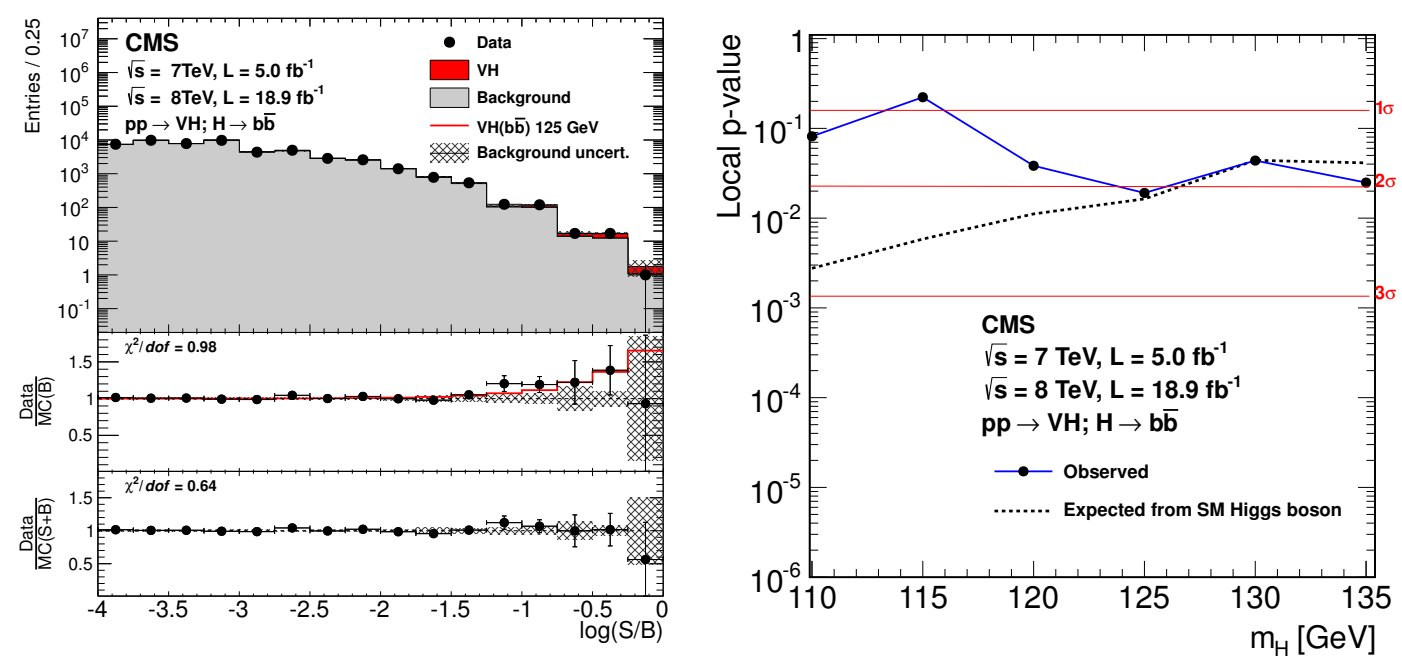

Figure 5. $\log (\mathrm{S} / \mathrm{B})$ distribution for events based on the output of their corresponding BDT discriminant (left), observed and expected significance distribution for the Higgs boson to a pair of b-jet produced in association with a $\mathrm{W}$ or a $\mathrm{Z}$ boson (right).

\section{9 ttH-production analyses}

Higgs boson production in association with a t $\bar{t}$ has one of the smallest cross-sections among possible production modes. However due to the rich final state with the presence of several leptons, missing $\mathrm{E}_{\mathrm{T}}$, jets and b-jets it is of great importance. Additionally this is the main production mode which tests the coupling of the Higgs boson to top quarks directly at the tree level. The search is performed in four channels, $\mathrm{H} \rightarrow \gamma \gamma, \mathrm{H} \rightarrow \tau \tau, \mathrm{H} \rightarrow \mathrm{bb}, \mathrm{H} \rightarrow$ multi-leptons. Several MVAs are trained to discriminate signal from backgrounds using information related to object kinematics, event shape, and the discriminant output from the b quark jet tagging algorithm [9].

\section{Higgs Properties}

A precise measurement of the Higgs boson mass is performed using the two high resolution channels, $\mathrm{H} \rightarrow \gamma \gamma$ and $\mathrm{H} \rightarrow \mathrm{ZZ}$. The mass resolution in each of these channels is about $1 \%$. Figure 6-left shows the $2 \mathrm{D}$ contour of the signal strength relative to the SM, $\mu=\sigma / \sigma_{\mathrm{SM}}$ and mass, $m_{\mathrm{H}}$ for the aforementioned final states. The combined curve is measured assuming the overall signal strength is a free parameter. The right plot shows the 1-D scan of the likelihood as a function of $m_{\mathrm{H}}$, where signal strengths relative to the $\mathrm{SM}, \mu_{\mathrm{ZZ}}, \mu_{\gamma \gamma}(\mathrm{ggH}, \mathrm{ttH})$ and $\mu_{\gamma \gamma}(\mathrm{VBF}, \mathrm{VH})$ are assumed to be independent and are left as free parameters. The mass is measured to be $m_{\mathrm{H}}=125.03_{-0.31}^{+0.29}$.

One of the simplest tests of the compatibility of the new observed state with the SM Higgs boson is the best fit value of the signal strength $\mu=\sigma / \sigma_{\mathrm{SM}}$. Figure 7-left shows this value for different production modes. A large deviation of the best fit value from 1 would be an indication of the incompatibility of the new state with the SM Higgs boson. As it is shown the best fit for all production mode tags are consistent with SM. A deviation in t⿱t$H$ channel is due to the excess seen in one of the final states in multi-lepton channel. The plot in the middle shows the best fit value in different decay modes. The combined value of the signal strength is measured to be $\mu=1.00 \pm 0.13$ which verifies 

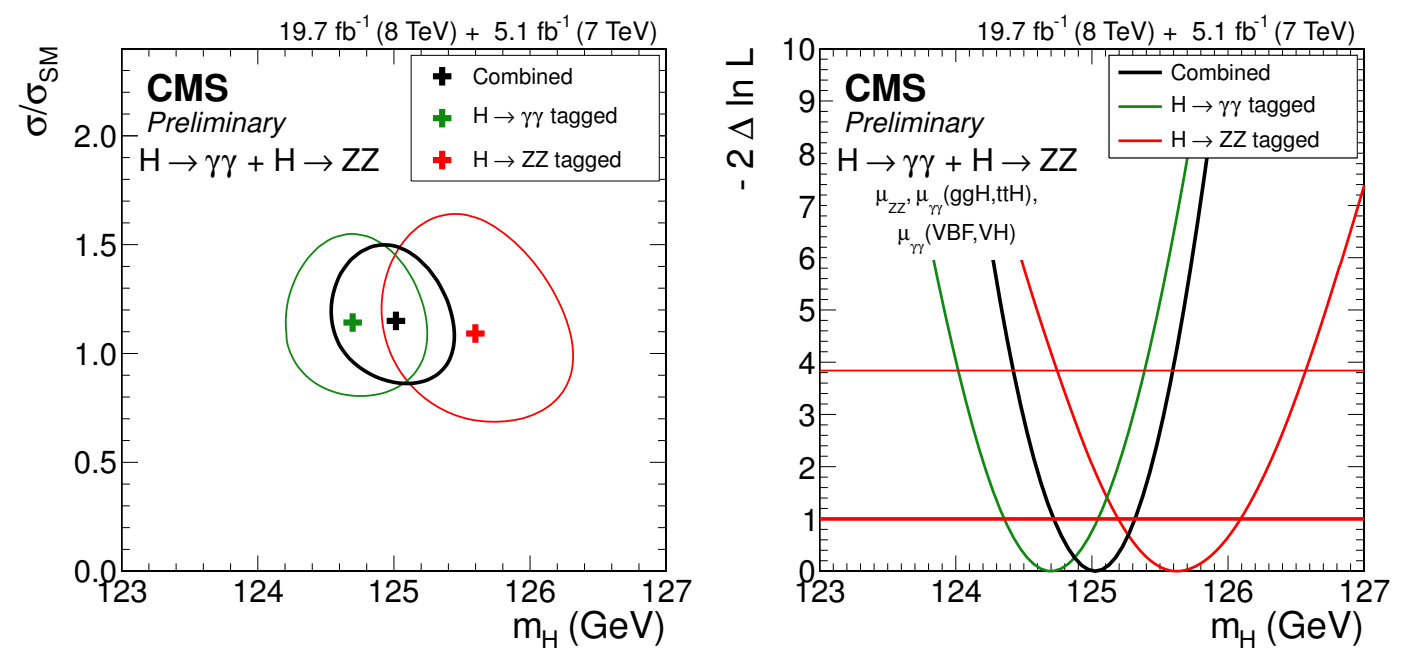

Figure 6. 2-D likelihood scan for 2 high resolution channels, $\mathrm{H} \rightarrow \gamma \gamma$ and $\mathrm{H} \rightarrow \mathrm{ZZ}$, in terms of the signal strength and Higgs mass (left), 1-D scan of the likelihood as function of $m_{\mathrm{H}}$ (right).

that the new observed state is compatible with the SM Higgs boson. Another test is to measure the signal strength of the vector boson related production processes (VBF and $\mathrm{VH}$ ) versus fermion related one $(\mathrm{ggH}$ and $\mathrm{t} \overline{\mathrm{H}})$ in each decay mode. These results are shown in figure 7-right, where $2 \mathrm{D}$ contours for different decay channels are shown in terms of the $\mu_{\mathrm{VBF}, \mathrm{VH}}$ and $\mu_{\mathrm{ggH}, \mathrm{ttH}}$. The crosses indicate the best-fit values obtained in each group of predominant decay modes: $\gamma \gamma, \mathrm{ZZ}, \mathrm{WW}, \tau \tau$, and bb. The diamond at $(1,1)$ indicates the expected values for the SM Higgs boson.
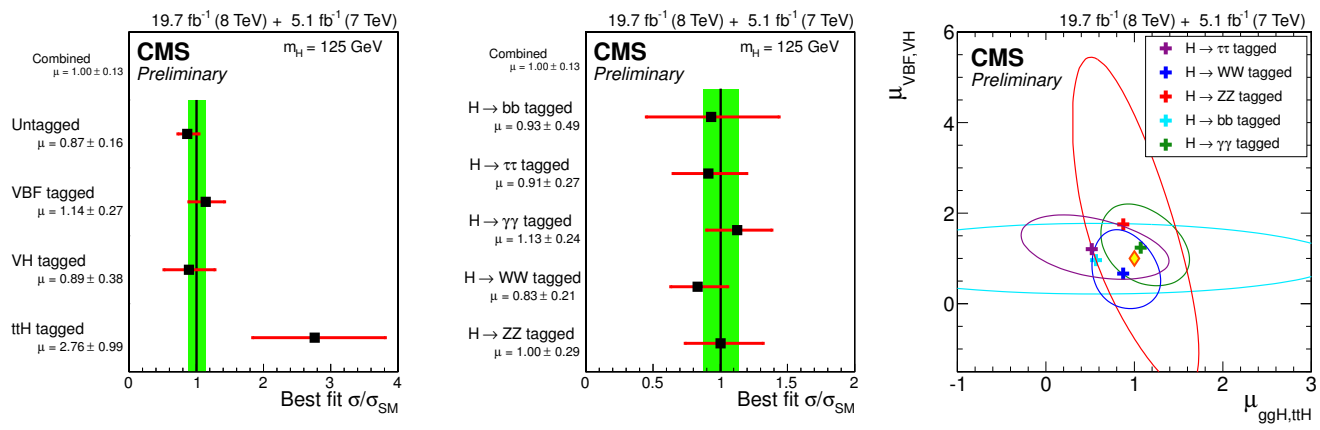

Figure 7. Best fit for different production modes(left), individual decay modes(middle) and combined VBF and VH v.s. combined $\mathrm{ggH}$ and $\mathrm{ttH}$ for different decay modes(right).

Another test of the compatibility of the observed data with the SM Higgs boson are the coupling measurements. Based on some assumptions on the spin-parity of the observed $\operatorname{state}\left(\mathrm{J}^{\mathrm{PC}}=0^{++}\right)$ and the narrow width approximation, production and decay of the Higgs boson can be considered 
independent. Consequently for any production and decay the following equation is valid:

$$
(\sigma \cdot B)(x \rightarrow \mathrm{H} \rightarrow f f)=\frac{\sigma_{x} \cdot \Gamma_{f f}}{\Gamma_{\text {tot }}}
$$

where $\sigma_{x}$ is the production cross section for the initial state $\mathrm{x}$ (including ggH, $\mathrm{VBF}, \mathrm{VH}$, and ttH), $\Gamma_{f f}$ is the partial decay width into the final state $\mathrm{ff}$ and $\Gamma_{\text {tot }}$ is the total width of the Higgs boson. To test the possible deviation of the different couplings to the standard model, new parameters called coupling modifiers, denoted by scale factors $\kappa_{i}$, are introduced. A different measured value of the $\kappa_{i}$ from unity is a hint of a deviation from the standard model. A test is performed to check the coupling modifiers of the fermions, $\kappa_{f}$ v.s. coupling modifiers of the vector bosons $\kappa_{V}$. Results of 2D likelihood scans for the $\kappa_{V}$ v.s. $\kappa_{f}$ is shown in figure 8-left. According to this plot, the SM value which is $(1,1)$ indicated a by a diamond is within 1 sigma of the best fit. The plot on the right shows the $68 \% \mathrm{CL}$ contours for different channels and for the combination for $\left(\kappa_{V}, \kappa_{f}\right)$ parameters.
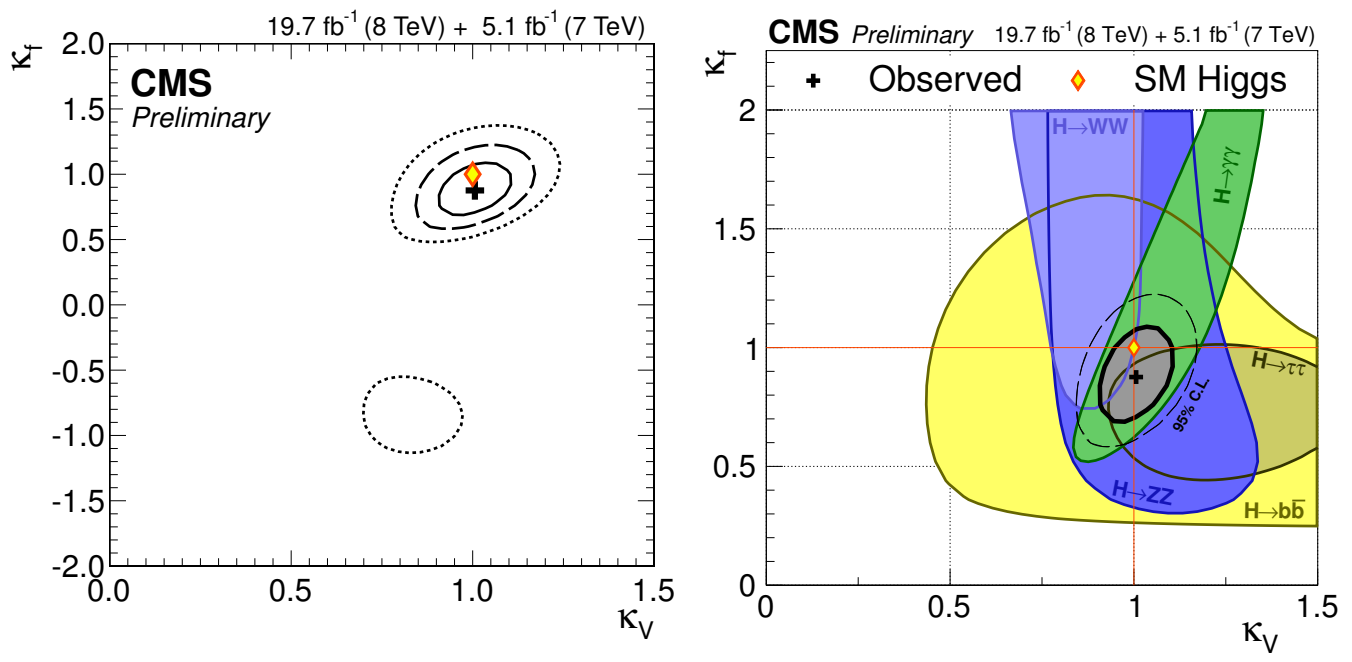

Figure 8. Results of 2D likelihood scans for the $\kappa_{V}$ v.s. $\kappa_{f}$ (left) and 68\% CL contours for different channels and for the combination for $\left(\kappa_{V}, \kappa_{f}\right)$ parameters (right).

Further tests have been performed to check the compatibility of the observed data with the SM Higgs boson in different models. They are summarised in figure $9 . \kappa_{V}$ and $\kappa_{f}$ are just discussed above. $\lambda_{\mathrm{WZ}}$ is defined as a ratio of the coupling to the $\mathrm{W}$ boson over the coupling to the $\mathrm{Z}$ boson, namely $\kappa_{W}$ and $\kappa_{Z}$, respectively. This measurement is a useful test for custodial symmetry which is a symmetry that protects the ratio between the masses and couplings of the $\mathrm{W}$ and $\mathrm{Z}$ boson, against large radiative corrections. A large violation of the custodial symmetry is a hint for the new physics model. As it is shown, $\lambda_{\mathrm{WZ}}$ is compatible with unity within one standard deviation. The next test is to check the ratio of the Higgs boson coupling to down-type fermions relative to the up-type fermions. Different couplings to up and down type fermions are highly motivated in models with multiple Higgs doublets, like Two Higgs Doublet Models or a supersymmetric extension of the Standard Model. According to the plots, no significant deviation is seen from unity. The other test indicated by $\lambda_{\mathrm{lq}}$ is targeted to test the deviation of the coupling to the leptons from quarks which is also compatible with unity within the error. As couplings to gluon and photon occur via loops, any deviation of the couplings 
from the SM values, would be an indirect implication of the existence of new physics which would manifest itself as new particles propagating in the loops. Measured values of $\kappa_{g}$ and $\kappa_{\gamma}$ shows no deviation from 1 , although the large uncertainties still leave some room for new physics. Finally a test is performed to set limits on the partial width of the beyond standard model $\Gamma_{\mathrm{BSM}}$. A likelihood scan versus $\mathrm{BR}_{\mathrm{BSM}}=\Gamma_{\mathrm{BSM}} / \Gamma_{\text {tot }}$, where $\kappa_{g}$ and $\kappa_{\gamma}$ are profiled together with other nuisance parameters, leads to the interval of $[0.00,0.32]$ at $95 \% \mathrm{CL}$ for the $\mathrm{BR}_{\mathrm{BSM}}$. This is indicated by the last line in the figure 9 [10].

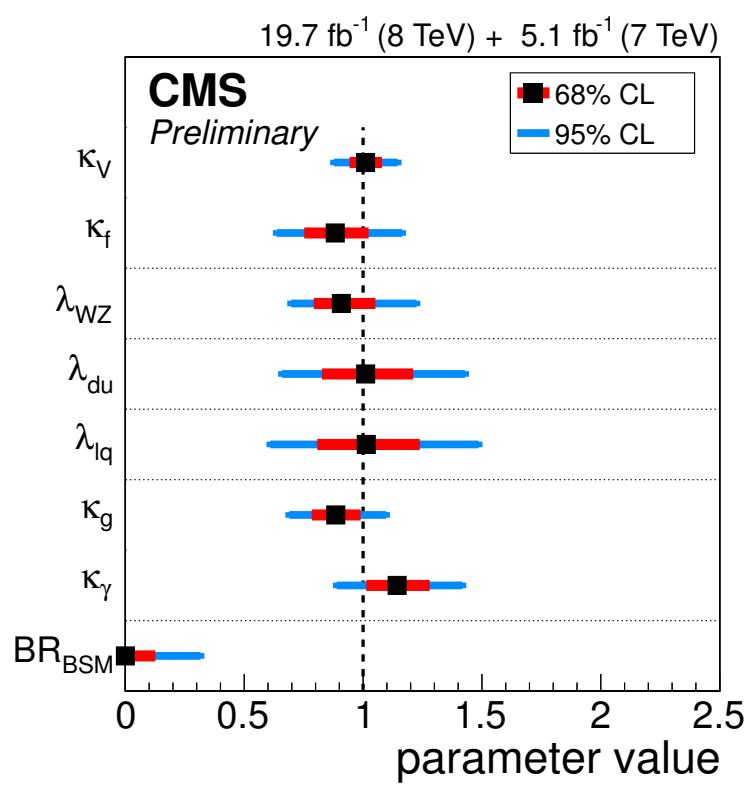

Figure 9. Summary plot of likelihood scan results for the different models separated by dotted lines. The inner bars represent the $68 \%$ CL confidence intervals while the outer bars represent the 95\% CL confidence intervals.

\section{References}

[1] CMS Collaboration, Phys. Lett. B 716, 30 (2012)

[2] ATLAS Collaboration, Phys. Lett. B 716 , 1 (2012)

[3] CMS Collaboration, JINST 3, S08004 (2008)

[4] CMS Collaboration, Eur. Phys. J. C 74, 3076 (2014)

[5] CMS Collaboration, Phys. Rev. D 89, 092007 (2014)

[6] CMS Collaboration, J. High Energy Phys. 01, 096 (2014)

[7] CMS Collaboration, J. High Energy Phys. 05, 104 (2014)

[8] CMS Collaboration, Phys. Rev. D 89, 012003 (2014)

[9] CMS Collaboration, J. High Energy Phys. 05, 145 (2013)

[10] CMS Collaboration, arXiv:1412.8662 (submitted to Eur. Phys. J. C.) (2014) 